\title{
A VARIATIONAL PRINCIPLE FOR SURFACE WAVES IN MAGNETOHYDRODYNAMICS*
}

\author{
By BHIMSEN K. SHIVAMOGGI (The Australian National University, Canberra)
}

\begin{abstract}
A variational principle for the motion of the interface between an infinitely conducting fluid and a vacuum magnetic field is given. This variational principle not only gives the Laplace equation governing the velocity potential and the magnetic-field potential, but also provides all the boundary conditions appropriate to the interface between an infinitely conducting fluid and a vacuum magnetic field. However, unlike the hydrodynamical case, this variational principle does not have the simple physical interpretation of the stationarity of the fluid pressure + magnetic field pressure.

An expression for the pressure was used by Bateman [1] as the Lagrangian and the equations of motion in an inviscid, incompressible fluid were derived from a variational principle. Luke [4] showed that a simple extension of this variational principle also provides the boundary conditions appropriate to a free surface. Debnath $[2,3]$ reformulated the full set of equations of motion for the surface-wave problem in magnetohydrodynamics, and made an initial-value investigation of the two- and three-dimensional dispersive surface waves in an electrically conducting fluid with a free surface. It is of special interest to consider a variational principle for the two-dimensional surface waves in magnetohydrodynamics, and to derive the full set of equations of motion used by Debnath [2] and Shivamoggi [5]. The purpose of this note is to give a variational principle for the motion of the interface between an infinitely conducting fluid and a vacuum magnetic field. This variational principle not only gives the Laplace equation governing the velocity potential and the magnetic-field potential, but also provides all the boundary conditions appropriate to the interface between an infinitely conducting fluid and a vacuum magnetic field. However, unlike the hydrodynamical case, this variational principle does not have the simple physical interpretation of the stationarity of the fluid pressure + magnetic field pressure.
\end{abstract}

Consider an infinitely-conducting fluid of density $\rho$ subjected to a gravitational field $\mathbf{g}=-g \hat{\mathbf{v}}_{y}$ and confined in the region $0<y<h$ by a vacuum magnetic field $\mathbf{H}$. Then the variational principle is

$$
\delta J=\delta \int_{t_{1}}^{t_{2}} \int_{x_{1}}^{x_{2}} L d x d t=0
$$

where

$$
L=\int_{0}^{h(x, t)}\left(\frac{1}{2} \phi_{x}^{2}+\frac{1}{2} \phi_{y}^{2}+\phi_{t}+g y\right) d y-\int_{h(x, t)}^{\infty} \frac{1}{\rho}\left(\frac{1}{2} \psi_{2}^{2}+\frac{1}{2} \psi_{y}^{2}\right) d y,
$$

\footnotetext{
* Received February 23, 1982; revised version received July 9, 1982. Author's present address: Physical Research Laboratory, Ahmedabad 380009, India.
} 
where $\phi(x, y, t)$ is the velocity potential describing the motion of the fluid, $\psi(x, y, t)$ the magnetic-field potential (i.e., $\mathbf{H}=\nabla \psi)$ and $\phi(x, y, t), \psi(x, y, t)$ and $h(x, t)$ are allowed to vary subject to the restrictions $\delta \phi=0, \delta \psi=0, \delta h=0$ at $x_{1}, x_{2}, t_{1}, t_{2}$.

Following the usual procedure in the calculus of variations, (1) gives, for small changes $\delta \phi, \delta \psi$ and $\delta h$ in $\phi, \psi$ and $h$, respectively,

$$
\begin{aligned}
\delta J= & \int_{t_{1}}^{t_{2}} \int_{x_{1}}^{x_{2}}\left\{\left[\frac{1}{2} \phi_{x}^{2}+\frac{1}{2} \phi_{y}^{2}+\frac{1}{\rho}\left(\frac{1}{2} \psi_{x}^{2}+\frac{1}{2} \psi_{y}^{2}\right)+\phi_{t}+g y\right]_{y=h} \delta h\right. \\
& +\int_{0}^{h(x, t)}\left(\phi_{x} \delta \phi_{x}+\phi_{y} \delta \phi_{y}+\delta \phi_{t}\right) d y \\
& \left.-\int_{h(x, t)}^{\infty} \frac{1}{\rho}\left(\psi_{x} \delta \psi_{x}+\psi_{y} \delta \psi_{y}\right) d y\right\} d x d t=0 .
\end{aligned}
$$

Upon integrating by parts, (3) gives

$$
\begin{aligned}
\delta J= & \int_{t_{1}}^{t_{2}} \int_{x_{1}}^{x_{2}}\left\{\left[\frac{1}{2} \phi_{x}^{2}+\frac{1}{2} \phi_{y}^{2}+\frac{1}{\rho}\left(\psi_{x}^{2}+\psi_{y}^{2}\right)+\phi_{t}+g y\right]_{y=h} \delta h\right. \\
& +\left[\left(-h_{x} \phi_{x}+\phi_{y}-h_{t}\right) \delta \phi\right]_{y=h}-\left[\phi_{y} \delta \phi\right]_{y=0} \\
& +\left[\frac{1}{\rho}\left(-h_{x} \psi_{x}+\psi_{y}\right) \delta \psi\right]_{y=h}-\left[\psi_{y} \delta \psi\right]_{y=\infty} \\
& \left.-\int_{0}^{h(x, t)}\left(\phi_{x x}+\phi_{y y}\right) \delta \phi d y+\int_{h(x, t)}^{\infty}\left(\psi_{x x}+\psi_{y y}\right) \delta \psi d y\right\} d x d t=0
\end{aligned}
$$

First let us choose

$$
\delta h=0, \quad[\delta \phi]_{y=0, h}=0, \quad[\delta \psi]_{y=h, \infty}=0 ;
$$

since $\delta \phi$ and $\delta \psi$ are arbitrary otherwise, one deduces from (4),

$$
\phi_{x x}+\phi_{y y}=0, \quad \psi_{x x}+\psi_{y y}=0 .
$$

Then, since $\delta h,[\delta \phi]_{y=0},[\delta \phi]_{y=h},[\delta \psi]_{y=h}$, and $[\delta \psi]_{y=\infty}$ may be given arbitrary independent values, (4) gives

$$
\begin{aligned}
& 0<y<h \text { : } \\
& \phi_{x x}+\phi_{y y}=0, \\
& y>h \text { : } \\
& \psi_{x x}+\psi_{y y}=0 \text {, } \\
& y=h: \\
& -h_{x} \phi_{x}+\phi_{y}-h_{t}=0 \text {, } \\
& y=h: \\
& -h_{x} \psi_{x}+\psi_{y}=0 \text {, } \\
& y=h: \\
& \frac{1}{2} \phi_{x}^{2}+\frac{1}{2} \phi_{y}^{2}+\frac{1}{\rho}\left(\frac{1}{2} \psi_{x}^{2}+\frac{1}{2} \psi_{y}^{2}\right)+\phi_{t}+g y=0, \\
& y=0 \text { : } \\
& \phi_{y}=0 \text {, } \\
& y=\infty: \\
& \psi_{y}=0 .
\end{aligned}
$$

(7) describes the kinematic condition on the velocity field at the interface. (8) describes the constraint of frozen-in lines of force in the infinitely conducting fluid on the vacuum magnetic field. (9) describes the force balance at the interface. (10) describes the condition 
of impenetrability of the fluid at the boundary $y=0$. (11) describes the fact the vacuum magnetic field far away from the interface must be aligned with the interface in order that the interface remain in static equilibrium in the presence of the vacuum magnetic field. This together with (8) implies that the interface remains a magnetic field line even in a perturbed state.

Note, however, that this variational principle, unlike the one in the hydrodynamical case, does not have the physical interpretation of the stationarity of the fluid pressure + magnetic field pressure.

The system of equations (5)-(11) has been used by Debnath [2] and Shivamoggi [5] to study linearized surface waves in magnetohydrodynamics.

\section{REFERENCES}

[1] H. Bateman, Partial differential equations, Cambridge Univ. Press, 1944

[2] L. Debnath, Plasma Phys. 19, 263 (1977)

[3] L. Debnath, Plasma Phys. 20, 343 (1978)

[4] J. C. Luke, J. Fluid Mech. 27, 395 (1967)

[5] B. K. Shivamoggi, J. Plasma Phys., 27, 321 (1982) 